\title{
Commentary on: delayed enhancement imaging of myocardial viability: low-dose high-pitch CT versus MRI
}

\author{
Florian Wolf
}

Received: 26 April 2011 / Accepted: 29 April 2011 /Published online: 15 May 2011

(C) European Society of Radiology 2011

In 2005, Nikolaou et al. [1] published a paper in European Radiology about the assessment of myocardial perfusion and viability from routine contrast-enhanced 16-detectorrow computed tomography. The authors reported promising results, however, concluded carefully: “... application of delayed enhancement techniques might be useful. ... However, this procedure requires a second delayed $C T$ scan, increasing the radiation exposure of the patient."

I remember the first scientific presentations about cardiac perfusion imaging and delayed enhancement using computed tomography - it was interesting, but the scientific community considered this topic more or less a scientific gimmick rather than serious work with clinical relevance.

Now, in 2011, Alkadhi et al. [2] publish in European Radiology a paper entitled "Delayed enhancement imaging of myocardial viability: Low-dose high-pitch CT versus MRI." The authors included 24 patients and could show that by using the newest generation CT equipment, delayed enhancement by CT has become a reality at a ultra-low radiation dose in submillisievert range, and is now ready for clinical use: The estimated mean radiation dose was as low as $0.89 \pm 0.07 \mathrm{mSv}$ (range: $0.74-1.02 \mathrm{mSv}$ ). This low radiation dose was obtained by using the "high-pitch" spiral CT technique with a pitch factor of 3.4 and prospective ECG-synchronization; furthermore a $100 \mathrm{kV}$ tube voltage was applied for the delayed enhancement CT data acquisition, making use of the higher attenuation of iodine at lower photon energies. In the present study, the

\section{F. Wolf $(\triangle)$}

Department of Radiology, Division of Cardiovascular and Interventional Radiology, Medical University of Vienna, Waehringer Guertel 18-20,

1090 Vienna, Austria

e-mail: florian.wolf@meduniwien.ac.at authors perform a standard contrast-enhanced CTA using a high-pitch protocol on a commercially available 128-slice, dual-source CT system after the administration of $70 \mathrm{ml}$ iodinated contrast media. In order to achieve an adequate dose of iodine, $5 \mathrm{~min}$ after the first CT data-acquisition, a second dose of $70 \mathrm{ml}$ of iodinated contrast media was administered and, after a total delay of around $15 \mathrm{~min}$ from the first injection, a second high-pitch delayed enhancement CT data-set was obtained. The sensitivity, specificity, and accuracy of CT for the diagnosis of non-viability were $90.0 \%, 92.9 \%$, and $91.7 \%$ per patient and $60.7 \%, 96.8 \%$ and $94.4 \%$ per segment, respectively, with MRI serving as the gold standard.

What happened in the last 6 years to make the vision of myocardial viability imaging a reality?

In my opinion, new technical developments such as lowdose gating techniques, prospective ECG-triggering and high-pitch CT are all responsible for this present step forward in cardiac imaging. The Cardiac Imaging 'hype' started with the introduction of 64-slice CT with a revolution in image quality. This first wave of excitement was followed by a disillusion: the radiation dose of cardiac CT reached values of $12-14 \mathrm{mSv}$, even higher than the radiation dose values of a conventional invasive angiography. However with the introduction of newer CT equipment with 128-, 256-, and 320-slices, as well as dual source CT-machines, faster CT protocols with radically lower radiation dose have become possible. In the present paper, the CTA-, data-acquisition, as well as the delayed enhancement imaging, is performed using a high pitch of up to 3.4, compared with $0.2-0.4$ for conventional normal retrospective ECG-triggered CT. Excellent image quality with high pitch has become possible due to recently introduced 128-dual-source CT technology. With this technique, low-dose $\mathrm{CT}$ is possible, with a radiation dose lower 
than $1 \mathrm{mSv}[3,4]$-not only just for scientific purposes, but also for everyday, routine clinical practice.

Several important papers about myocardial viability imaging and particularly the evaluation of late-enhancement by CT have been published over the last several years in European Radiology as well as in other important imaging journals. In 2007, Mahnken et al. [5] published a very important paper about late-phase multidetector CT (MDCT) in different stages of myocardial infarction in a pig model. They found no significant difference between MDCT and MR imaging for all different stages of myocardial infarction, as well as a good correlation between infarct size at histopathology and both imaging methods.

In 2010, Mahnken et al. [6] tried to find the culprit lesion by combined visualization of the coronary arteries and delayed myocardial enhancement in dual-source CT. According to the authors, the combined approach was successful, with a good automated assessment of myocardial infarction size from late-phase CT.

Beginning in 2005, different authors [1, 7-9] attempted perfusion imaging with $\mathrm{CT}$ under resting conditions to evaluate the density of the myocardium in normal CTA or in test bolus data acquisitions [10]. All these authors found good correlation, with nuclear medicine or MRI serving as the gold standard.

In 2008 Cury et al. [11] validated CT first-pass perfusion imaging under resting conditions combined with wall motion and left ventricular function with SPECT serving as gold standard. Blankstein et al. [12] validated CT perfusion imaging under adenosine stress against myocardial SPECT and conventional invasive angiography and found a sensitivity of $79 \%$ and specificity of $80 \%$ for detection of stenosis $>50 \%$ by CT perfusion alone on a pervessel-basis. But, radiation dose matters, and radiation dose using 64-slice CT was high with $13.7 \pm 3.4 \mathrm{mSv}$ for single [11] and 9.1 $\pm 3.9 \mathrm{mSv}$ for dual source CT [12], hence the justification of a second delayed enhancement CT dataacquisition became questionable. Moreover, image noise by CT is significantly higher than CMR [10]. In my opinion, CMR should be preferred for viability imaging "stand alone", as it is radiation free and provides better image quality. However, in patients in whom additional evaluation of coronary (or bypass graft) vessel anatomy is required, $\mathrm{CT}$ is a reasonable alternative for a combined coronary CTA and viability study.

Similarly, George et al. [13] validated adenosine stress perfusion in combination with $\mathrm{CT}$ angiography by comparing it with conventional angiography and SPECT, serving as gold standard. They found on a per vessel/territory basis a sensitivity of $79 \%$ and a specificity of $91 \%$.

Rocha et al. [14] observed incremental value of CTperfusion over CT-angiography to define hemodynamic relevance of a coronary stenosis.
Recently, a paper was published in European Radiology by Ko et al. [15], which compared adenosine stress perfusion dual-energy CT and CTA of the coronary arteries with stress-perfusion MRI and conventional coronary angiography; they showed that adenosine-stress dualenergy-CT can identify perfusion defects with a sensitivity, specificity and accuracy of $89 \%, 78 \%$, and $82 \%$, respectively. But, at the moment, the radiation dose from such combined protocols is still too high to use in the daily clinical routine.

The present paper by Alkadhi et al. [2] shows that delayed enhancement is ready to be used in routine clinical practice, given its remarkable low radiation dose. Adenosine stress-perfusion imaging [11-15] is feasible and several studies show promising results. The clear advantage of $\mathrm{CT}$ in this setting is the complementary evaluation of coronary artery anatomy and myocardial function, which overcomes current shortcomings of CTA.

The vision of the present and upcoming scientific work is to achieve a "one-stop-shop" encompassing both coronary morphology and myocardial function in cardiac imaging. And we are quite close to the goal-line for this scientific process.

\section{References}

1. Nikolaou K, Sanz J, Poon M et al (2005) Assessment of myocardial perfusion and viability from routine contrastenhanced 16-detector-row computed tomography of the heart: preliminary results. Eur Radiol 15:864-871

2. Alkadhi H, Goetti R, Feuchtner G et al (2011) Delayed enhancement imaging of myocardial viability: low-dose high-pitch CT versus MRI. Eur Radiol. doi:10.1007/s00330-011-2149-8

3. Lell M, Marwan M, Schepis T et al (2009) Prospectively ECGtriggered high-pitch spiral acquisition for coronary $\mathrm{CT}$ angiography using dual source CT: technique and initial experience. Eur Radiol 19:2576-2583

4. Leschka S, Stolzmann P, Desbiolles L et al (2009) Diagnostic accuracy of high-pitch dual-source CT for the assessment of coronary stenoses: first experience. Eur Radiol 19:2896-903, Epub 2009 Sep 16

5. Mahnken AH, Bruners P, Muhlenbruch G et al (2007) Low tube voltage improves computed tomography imaging of delayed myocardial contrast enhancement in an experimental acute myocardial infarction model. Invest Radiol 42:123-129

6. Mahnken AH, Bruners P, Friman O, Hennemuth A (2010) The culprit lesion and its consequences: combined visualization of the coronary arteries and delayed myocardial enhancement in dualsource CT: a pilot study. Eur Radiol 20:2834-2843

7. Kachenoura N, Lodato JA, Gaspar T et al (2009) Value of multidetector computed tomography evaluation of myocardial perfusion in the assessment of ischemic heart disease: comparison with nuclear perfusion imaging. Eur Radiol 19:18971905

8. Kachenoura N, Veronesi F, Lodato JA et al (2010) Volumetric quantification of myocardial perfusion using analysis of multidetector computed tomography 3D datasets: comparison with nuclear perfusion imaging. Eur Radiol 20:337-347 
9. Schepis T, Achenbach S, Marwan M et al (2010) Prevalence of first-pass myocardial perfusion defects detected by contrastenhanced dual-source CT in patients with non-ST segment elevation acute coronary syndromes. Eur Radiol 20:1607-1614

10. Groves AM, Goh V, Rajasekharan S et al (2008) CT coronary angiography: quantitative assessment of myocardial perfusion using test bolus data-initial experience. Eur Radiol 18:2155-2163

11. Cury RC, Nieman K, Shapiro MD et al (2008) Comprehensive assessment of myocardial perfusion defects, regional wall motion, and left ventricular function by using 64-section multidetector CT. Radiology 248:466-475

12. Blankstein R, Shturman LD, Rogers IS et al (2009) Adenosineinduced stress myocardial perfusion imaging using dual-source cardiac computed tomography. J Am Coll Cardiol 54:1072-1084
13. George RT, Arbab-Zadeh A, Miller JM et al (2009) Adenosine stress 64- and 256-row detector computed tomography angiography and perfusion imaging: a pilot study evaluating the transmural extent of perfusion abnormalities to predict atherosclerosis causing myocardial ischemia. Circ Cardiovasc Imaging 2:174-182

14. Rocha-Filho JA, Blankstein R, Shturman LD et al (2010) Incremental value of adenosine-induced stress myocardial perfusion imaging with dual-source CT at cardiac CT angiography. Radiology 254:410-419

15. Ko SM, Choi JW, Song MG et al (2011) Myocardial perfusion imaging using adenosine-induced stress dual-energy computed tomography of the heart: comparison with cardiac magnetic resonance imaging and conventional coronary angiography. Eur Radiol 21:26-35 